\title{
Efficacy of two Different Regimens Regarding Urinary Catheter Removal Time after Elective Cesarean Section: A Prospective Study
}

\author{
Magdy R. Ahmed and Ahmed A. Abu-Elrose
}

Department of Obstetrics and Gynecology, Faculty of Medicine, Suez Canal University, Egypt

\begin{abstract}
Background: The proper time for removing the urinary catheter after caesarian section (CS) is still controversial and the decision of when to remove the catheter postoperatively is based on custom rather than evidence-based knowledge. Aim: To assess whether early (after $8 \mathrm{~h}$ ) or delayed (after $24 \mathrm{~h}$ ) removal of a urinary catheter after elective CS could affect, the rate of urinary retention with re-catheterization, rate of symptomatic urinary tract infections (UTI), ambulation time and hospital stay length. Materials\& Methods: Prospective randomized trial conducted at obstetrics and gynecology department of Suez Canal University Hospitals. One hundred and fifty-one women underwent for term elective CS and were randomly allocated into two groups by simple randomization method. Women in group A (73 patients) had their urinary catheter removed $8 \mathrm{~h}$ post-operatively while in group $B$ (67 patients) the catheter was removed after 24 h. The following outcomes were assessed: rate of re-catheterization, symptomatic UTI, ambulation time and hospital stay length. Results: Regarding the incidence of urinary retention and recatheterization, patients in group A showed none significantly statistically difference when compared to patients in group B (2.7\% versus $0 \%$ respectively). Delayed urinary catheter removal was associated with a higher incidence of UTI (16.4\%), delayed ambulation time (11.3 h) and longer hospital stay (3.1days) compared to the early removal group (4.1\%, $6.7 \mathrm{~h}$ and 1.8 days respectively). Conclusion: Removal of the urinary catheter $8 \mathrm{~h}$ postoperatively appears to be more advantageous than late removal in cases of elective CS.
\end{abstract}

Key words: Urinary catheter; Caesarian Section; Urinary Tract infections

\section{Introduction}

Cesarean section (CS) is considered as an international phenomenon as rates have been increasing all over the world. Egypt is not an exception with a recent report showing caesarian section rate of $51.8 \%$ which is a way higher than that recommended by the World Health Organization $^{(1)}$. Insertion of urinary catheter is a preparatory step in CS as keeping the urinary bladder continues drained can improve uterine exposure, prevent iatrogenic urinary bladder injury and help the surgical team to assess the urinary output and to be assured that the urine appears clear ${ }^{(2,3)}$. Traditionally speaking, after CS the catheter is left for 24 hours to prevent postoperative urinary retention which can cause bladder atony, with long-term uri- 
nary sequel as voiding difficulties and detrusor over activity ${ }^{(2,4)}$. On the other hand, prolonged insertion of urinary catheter may lead to delayed patient ambulation because of patients' discomfort and fear of accidental removal of catheter when moving., increased urine bacterial colonization with subsequent UTI leading to increased overall healthcare and economic burdens ${ }^{(5,6)}$. The expert panel of the Infectious Diseases Society of America agreed that there is strong evidence that urinary catheters should be removed as soon as they are no longer required. The proper time for removing the indwelling urethral catheter after CS is still controversial and the decision of when to remove the catheter postoperatively is based on custom rather than evidence-based knowledge $(2,7)$. Two large RCTs and one non-RCT, suggested that the routine use of urinary catheters for cesarean delivery is not necessary, and can be harmful. However, further trials were recommended to confirm these findings ${ }^{(8)}$. This has inspired us to conduct this study to assess whether the early ( $8 \mathrm{~h}$ ) or delayed ( $24 \mathrm{~h}$ ) removal of urinary catheter after elective CS can affect the rate of urinary retention with recatheterization, rate of symptomatic urinary tract infections, ambulation time and the length of hospital stay.

\section{Materials and Methods}

This study was carried out in accordance to the principles for medical research involving human subjects included in Helsinki declaration. This prospective randomized study was performed at obstetrics and gynecology department of Suez Canal University Hospitals, Ismailia, Egypt from the start of March 2017 to the end of July 2018. All women fulfilling the study criteria were counseled and informed written consent was obtained from all participants. We included women with term singleton pregnancy prepared for term elective CS either primary or repeated. Exclusion criteria were women who had urinary tract infections preoperatively confirmed by midstream urine analysis, women with iatrogenic bladder injury so that urinary catheter had to be kept postoperatively on the surgeon's decision, women with any medical conditions requiring postoperative monitoring of urinary output as preeclampsia and women who had spinal anesthesia or contraindicated for general anesthesia. A total of 151 patients underwent elective CS. Eleven patients were finally excluded from the study; four patients had intraoperative iatrogenic bladder injury, while seven patients did not complete the postoperative follow-up. The remaining 140 women were divided into 2 groups by simple randomization using computer-generated random numbers. Participants in group $A$ (73 patients) had their urinary catheter removed $8 \mathrm{~h}$ post-operatively while in group B (67 patients) the catheter was left for $24 \mathrm{~h}$ after $\mathrm{CS}$. The required sample size was calculated based on power of the study of $80 \%$ and $\alpha$-error of $0.05^{(9)}$. On the morning of CS, all patients received a single dose of prophylactic antibiotic in the form of ceftriaxone $1 \mathrm{~g}$ intramuscularly. A size 14 silicone, 2-way Foley's catheter was introduced under aseptic technique after induction of general anesthesia. CS was performed in the usual manner. Then, based on the study design, the catheter was removed after the surgical procedure, either at 8 or $24 \mathrm{~h}$ after the operation. We opted to follow post CS the strict urinary retention definition, as inability for spontaneous micturition within 6 hours after the removal of urinary catheter ${ }^{(10)}$. Hence, after urinary catheter removal in both study groups, they were subsequently encouraged for spontaneously empty their bladders. If they could not void spontaneously 6 hours after catheter removal and 
the bladder was full on clinical abdominal examination with palpable urinary bladder; a urinary catheterization with a sterile disposable female in-out catheter was carried out once ${ }^{(2)}$. Urinary catheter was inserted if the patient could not empty the bladder $12 \mathrm{~h}$ after catheter removal and/or there was a palpable distended urinary bladder by abdominal examination $(2,11)$. All patients had a midstream urine sample taken on the second postoperative day for urine analysis. In addition, a third sample of urine was obtained after one week of surgery. The following outcomes were assessed for each group: rate of recatheterization, symptomatic urinary tract infection, time of ambulation and length of hospital stay. The diagnosis of symptomatic urinary tract infection was based on the following criteria: significant bacteriuria with at least one of the following symptoms; dysuria, frequency of micturition, urgency, supra pubic pain or burning sensation at micturition. The time to ambulation was defined as the period between the end of surgery and the time when the patient first walked supported by a nurse or relative. The length of hospi- tal stay was defined as the time between the end of surgery and hospital discharge $^{(12)}$. Data were processed using SPSS version 16 (SPSS Inc., Chicago, IL, USA). Quantitative data were expressed as mean \pm SD while qualitative data were expressed as numbers and percentages (\%). Student's t-test and the F-test were used to test the significant difference for quantitative variables while the Chi-square and Fisher's exact tests were used to test the significance for qualitative variables. $A$ probability value ( $p$-value) $<0.05$ was considered statistically significant.

\section{Results}

Baseline maternal characteristics at cesarean delivery in all groups are presented in Table 1. All groups were matched regarding maternal age, body mass index, gestational age at delivery, duration of surgery and indications for CS. Of note, the most common indication for CS was found to be previous CS, which was the indication for more than half of all patients $(57.4 \%$ and $59.7 \%$ in both groups $A$ and $B$ respectively) (Table 1).

Table 1: Baseline characteristics of the study groups

\begin{tabular}{|c|c|c|c|c|c|}
\hline & \multicolumn{2}{|c|}{$\begin{array}{c}\text { Group A } \\
(\mathrm{n}=73)\end{array}$} & \multicolumn{2}{|c|}{$\begin{array}{c}\text { Group B } \\
(n=67)\end{array}$} & p-value \\
\hline Age (Years) Mean \pm SD & \multicolumn{2}{|c|}{$30.1 \pm 4$} & \multicolumn{2}{|c|}{$28.6 \pm 3$} & 0.5 (NS) \\
\hline $\mathrm{BMI}\left(\mathrm{Kg} / \mathrm{m}^{2}\right)$ Mean $\pm \mathrm{SD}$ & \multicolumn{2}{|c|}{$28.5 \pm 2.7$} & \multicolumn{2}{|c|}{$29.1 \pm 3.2$} & $0.2(\mathrm{NS})$ \\
\hline Gestational age (weeks) Mean \pm SD & \multicolumn{2}{|c|}{$38.9 \pm 1.1$} & \multicolumn{2}{|c|}{$38.3 \pm 1.4$} & 0.5 (NS) \\
\hline \multicolumn{6}{|l|}{ Indications for CS no. (\%) } \\
\hline Previous CS & 42 & $(57.4 \%)$ & 40 & $(59.7 \%)$ & \multirow{4}{*}{0.6 (NS) } \\
\hline CPD & 19 & $(26.2 \%)$ & 13 & $(19.4 \%)$ & \\
\hline Mal-presentation & 8 & $(10.9 \%)$ & 9 & $(13.4 \%)$ & \\
\hline Maternal request & 4 & $(5.4 \%)$ & 5 & $(7.4 \%)$ & \\
\hline Duration of operation (min) Mean \pm SD & \multicolumn{2}{|c|}{$47.6 \pm 15 \cdot 3$} & \multicolumn{2}{|c|}{$49.4 \pm 13.4$} & 0.7 (NS) \\
\hline
\end{tabular}

NS = No statistically significant difference between both groups

$\mathrm{BMI}=$ body mass index; $\mathrm{CS}$ = cesarean section; $\mathrm{CPD}=$ cephalo-pelvic disproportion

The main outcome measures of the study are presented in (Table 2). Regarding the incidence of urinary retention and recatheterization, patients in group $A$ showed none significantly statistically dif- ference when compared to patients in group B (2.7\% versus $0 \%$ respectively). However, $16.4 \%$ of the studied patients in group B had symptoms suggestive of UTI as opposed to only $4.1 \%$ of patients in 
group A. Also, bacteriuria in urine samples received one day after catheter removal showed that $16.4 \%$ of patients in group B were positive versus $4.1 \%$ of patients in group A. It was found that patients in group $B$ had prolonged hospital stay with mean duration of 3.1 days, which is significantly longer than group A (1.8 days). Also, it was observed that patients in group $B$ had delayed first ambulation time $(11.3 \mathrm{~h}$ vs. $6.7 \mathrm{~h}$ in group $\mathrm{A})$. One week after removal of the catheter, it was found that
$16-22 \%$ of patients in group B had symptomatic lower UTI (dysuria, frequency and urgency) while $2.9 \%$ of the same group had symptoms suggestive of nephritis (fever and loin pain), whereas in group $A$ only two patients (1.3\%) showed symptoms suggestive of lower urinary tract infection only. Regarding postoperative bacteriuria, about $15 \%$ of patients in group $B$ were positive versus none of patients in group A (Table 3).

Table 2: Main outcome measures of the study groups

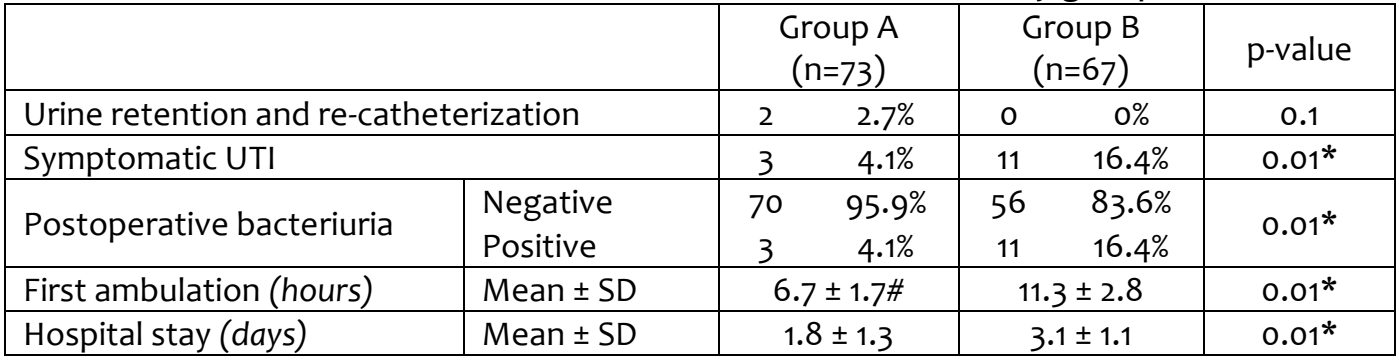

* Statistically significant difference between both groups

\section{Discussion}

Our study shows that removal of the urinary catheter $8 \mathrm{~h}$ after CS is associated with low rates of urinary retention (2.7\%), a finding is not observed in the delayed removal group. As the detrusor muscle functions independently of intra-abdominal pressure, with the passage of time after CS the ability to increase the intra abdominal pressure will be less affected by the painful surgical wound and can thus help the voiding process, which can explain the absence of urinary retention in the delayed removal group ${ }^{(2)}$. In agreement with our results, Chai and Pun confirmed that finding and that finding was not also significantly different, as their sample size was very small(13). El-Mazny and his colleges, did not found also any significant differences between early or delayed urinary catheter removals regarding the rate of urinary retention necessitating re-catheterization ${ }^{(11)}$. A lower incidence of urinary tract infections was ob- served in cases that underwent early removal of the catheter $(4.1 \%)$ in comparison to the delayed removal group (16.4\%). One week later, the delayed removal group had significantly higher rates of lower UTI symptoms with $15 \%$ of positive urinary bacteriuria; hence concurrent with our results many studies demonstrated that prolonged postoperative catheterization was considered the main triggering factor for $\mathrm{UTI}^{(2,13,14)}$. El-Mazny et al. in their study comparing immediate and delayed removal of urinary catheter after elective cesarean section; found that lower significant incidence of postoperative bacteriuria, dysuria, urinary frequency and urgency in the immediate group when compared with delayed removal group ${ }^{(11)}$. However, Onile and his group, found a nonsignificant lower incidence of UTI for women in the immediate removal group compared with those delayed removal after $24 \mathrm{~h}^{(15)}$. A Foley catheter in the bladder acts as a foreign body in a moist environment which enhances colonization of 
biofilm microorganisms that have the great ability to resist antimicrobial agents. This means that urinary urinary catheter cannot usually be cleared of a pathogenic biofilm without removing the catheter ${ }^{(16)}$. Unfortunately, those biofilm-associated organisms can seed the urine with bacteria with an increased risk of bacteriuria by 5-10\% per day of use of a urinary cathe$\operatorname{ter}^{(2,11)}$. Interestingly; ambulation time and hospital stay were found to be correlated to the timing of urinary catheter removal, as the delayed catheter removal group had a prolonged time of ambulation and hospital stay in comparison to the other group. The prolonged hospital stay could be explained by the presence of symptomatic UTI and positive bacteriuria cases which necessitated lengthy in patients with antibiotic treatment. Concurrent with our results, some studies demonstrated that finding $(2,11,17)$. As catheters might interfere with early ambulation from discomfort, pain and patient anxiety. According to fact that, early ambulation reduces bladder and bowel inertia, decreases the risk of DVT and improves respiratory function. Hence, early ambulation is therefore a recommended and encouraged in our practice.

Table 3: Urinary symptoms and urine analysis one week after CS

\begin{tabular}{|l|l|cc|cc|c|}
\hline \multicolumn{2}{|c|}{} & \multicolumn{2}{|c|}{ Group A (n=73) } & \multicolumn{2}{c|}{ Group B (n=67) } & p-value \\
\hline \multirow{3}{*}{ Symptoms } & Fever & 0 & $0 \%$ & 2 & $2.9 \%$ & $0.09(\mathrm{NS})$ \\
& Dysuria & 0 & $0 \%$ & 11 & $16.4 \%^{\#}$ & $0.001^{*}$ \\
& Frequency & 1 & $1.3 \%$ & 15 & $22.3 \%^{\#}$ & $0.002^{*}$ \\
& Urgency & 1 & $1.3 \%$ & 12 & $17.9 \%^{\#}$ & $0.001^{*}$ \\
& Loin pain & 0 & $0 \%$ & 2 & $2.9 \%$ & $0.09(\mathrm{NS})$ \\
\hline Postoperative & Negative & 73 & $100 \%$ & 57 & $85.1 \%{ }^{\#}$ & $0.001^{*}$ \\
bacteriuria & Positive & 0 & $0 \%$ & 10 & $14.9 \%$ & \\
\hline
\end{tabular}

NS: Not significant; * Statistically significant difference between both groups

\section{Conclusion}

Early removal of the urinary catheter had a lower incidence of urinary tract infection, earlier ambulation and shorter hospital stay. On the other hand, delayed urinary catheter removal was associated with higher incidence of urinary tract infection, delayed ambulation and longer hospital. Interestingly, urinary catheter removal just $8 \mathrm{~h}$ after CS appears to be more advantageous than late catheter removal in all our study outcomes.

\section{Acknowledgements}

We would like to thank our patients for participating in the study. We also appreciate the help of the nursing staff in the obstetrics department throughout the course of the study.

\section{References}

1- El-Zanaty and Associates. Ministry of health and population. Rockville (MD): The DHS Program ICF International. Egypt Demographic and Health Survey; 2014.

2- Ahmed MR, Ahmed WA, Attwa KA, et al. Timing of urinary catheter removal after uncomplicated total abdominal hysterectomy; a prospective randomized trial. Eur J Obstet Gynecol Reprod Biol..2014; 176:60-3.

3- Ghoreishi J. Indwelling urinary catheters in Cesarean delivery. Int J Gynecol Obstet 2003; 83:267-70.

4- Stanton SL, Ozsoy C, Hilton P. Voiding difficulties in the female: prevalence, clinical and urodynamic review. Obstet Gynecol J 1983; 61:144-7. 
5- Martinez OV, Civetta JM, Anderson K, et al. Bacteriuria in the catheterized surgical intensive care patient. Crit Care Med J 1986;14: 188-91.

6- Summitt RL, Stovall TG, Bran DF. Prospective comparison of in-dwelling bladder catheter drainage versus no catheter after vaginal hysterectomy. Am J Obstet Gynecol 1994; 170:181521.

7- Hooton TM, Bradley SF, Cardenas DD, et al. Diagnosis, prevention, and treatment of catheter-associated urinary tract infection in adults: 2009 international clinical practice guidelines from the infectious diseases society of America. Clin Infect Dis 2010;50 (5): 625-63

8- Li L, Wen J, Wang L, et al. Is routine indwelling catheterization of the bladder for caesarean section necessary? A systematic review. $\mathrm{Br} J$ Obstet Gynaecol 2011;118(4):400-9.

9- Fleiss JL. Statistical methods for rates and proportions. New York: John Wiley \& Sons; 1981

10- Yip SK, Sahota D, Pang MW, Chang A. Postpartum urinary retention. Acta Obstet Gynecol Scand 2004; 83 (10): 881-91.

11- El-Mazny A, El-Sharkawy M, Hassan A. A prospective randomized clinical trial comparing immediate versus delayed removal of urinary catheter following elective cesarean section .Eur J Obstet Gynecol Reprod Biol.2014; ;11-14.

12- Alessandri F, Mistrangelo E, Lijoi D, et al. A prospective, randomized trial comparing immediate versus delayed catheter removal following hysterectomy. Acta Obstet Gynecol Scand 2006;85: 716-20.

13- Chai J, and Pun TC. A prospective randomized trial to compare immediate and 24- hour delayed catheter removal following total abdominal hysterectomy. Acta Obstet Gynecol Scand 2011; 90:478-82.

14- Evron S, Dimitrochenko V, Khazin V, et al. The effect of intermittent versus continuous bladder catheterization on labor duration and postpartum urinary retention and infection: a randomized trial. J Clin Anesth 2008;20(8):567-72

15- Onile TG, Kuti O, Orji EO, et al. A prospective randomized clinical trial of urethral catheter removal following elective cesarean delivery. Int J Gynaecol Obstet 2008;102(3):267-70

16- Trautner BW, Hull RA, Darouiche RO. Prevention of catheter-associated urinary tract infection. Curr Opin Infect Dis 2005; 18:37-41.

17- Tambyah PA, Maki DG. Catheterassociated urinary tract infection is rarely symptomatic: a prospective study of 1,497 catheterized patients. Arch Intern Med 2000; 160(5):678-82. 